\section{Case Reports in Neurology}

Case Rep Neurol 2017;9:62-68

DOI: $10.1159 / 000466684$

Published onlıne: April 24, 2017

2017

This article is licensed under the Creative Commons Attribution-NonCommercial 4.0
International License (CC BY-NC) (http://www.karger.com/Services/OpenAccessLicense).

Usage and distribution for commercial purposes requires written permission.

\title{
Pure Amorphagnosia without Tactile Object Agnosia
}

\author{
Shinichirou Kubota ${ }^{a}$ Mai Yamada ${ }^{a}$ Hideyo Satoh $^{\mathrm{b}}$ Akira Satoh $^{\mathrm{b}}$ \\ Mitsuhiro Tsujihata ${ }^{b}$ \\ ${ }^{a}$ Section of Rehabilitation, Nagasaki Kita Hospital, Nagasaki, Japan; ${ }^{b}$ Section of \\ Neurology, Nagasaki Kita Hospital, Nagasaki, Japan
}

\section{Keywords}

Amorphagnosia $\cdot$ Tactile agnosia $\cdot$ Ahylognosia $\cdot$ Cerebral infarction

\begin{abstract}
A 54-year-old female showed amorphagnosia without ahylognosia and tactile agnosia 40 days after the onset of right cerebral infarction. Her basic somatosensory functions were normal. The appreciation of substance qualities (hylognosia) was preserved, but the patient's inability to recognize the size and shape (morphagnosia) was confined to 2- and 3dimensional shapes (amorphagnosia) in the left hand. However, the patient's ability to recognize real daily objects was well preserved. Brain MRI after admission showed ischemic lesions confined to the right pre- and postcentral gyri and the medial frontal cortex on DWI and FLAIR images. An analysis of SPECT images revealed that the most decreased areas were localized to the pre- and postcentral gyri, superior and inferior parietal lobules, supramarginal gyrus, and angular gyrus. Considering the previous reported cases, the responsible lesion for the impaired perception of hylognosia and morphagnosia may not necessarily be confined to the right hemisphere. To date, 5 reports ( 6 cases) of tactile agnosia have been published; 4 cases presented with both ahylognosia and amorphagnosia, while 1 presented with only amorphagnosia, and another showed amorphagnosia and mild ahylognosia. Our case is the first to present with only amorphagnosia without tactile agnosia. The mechanism for the well-preserved recognition of real objects may depend on the preserved hylognosia. Of note, there have been no reports showing only ahylognosia without amorphagnosia. Further studies are necessary to clarify whether or not patients with preserved hylognosia or morphagnosia retain the ability to perceive real objects.




\section{Introduction}

The concept of tactile agnosia refers to patients who are unable to recognize objects in one modality in the presence of relatively intact sensory functions and adequate cognition. Compared with visual agnosia, relatively few reports of tactile agnosia have been described in the literature.

In 1895, Wernicke [1] reported a case of sensory paralysis (Tastlaemung) with impairment of tactile recognition; however, the somatosensory deficits were disproportionately less severe than the recognition deficit. Wernicke distinguished disorders of primary identification, in which the patient is unable to recognize the tactile qualities of an object (apperceptive agnosia), from those of secondary identification, in which the recognition of an object's meaning is affected (associative agnosia).

In 1935, Delay [2] reported the concept of primary identification by introducing the distinction between hylognosia (recognition of the features related to the substance of the object) and morphagnosia (recognition of its size and shape).

We herein report a case suggestive of a pure form of amorphagnosia, in which the patient was not associated with ahylognosia and tactile agnosia.

\section{Case}

A 54-year-old female, with 12 years of schooling, noted the sudden onset of transient numbness and weakness of the left extremities in January 2012. She was diagnosed with suffering from moyamoya disease. On June 10, she again suffered from transient left hemiparesis. On July 20, she was admitted to our hospital for rehabilitation.

A neurological examination on admission revealed no abnormal findings. Brain magnetic resonance imaging (MRI) showed ischemic lesions confined to the right pre- and postcentral gyri and the medial frontal cortex on diffusion-weighted images (DWI) and fluidattenuated inversion recovery (FLAIR) images (Fig. 1a). An analysis of single-photon emission computed tomography (SPECT) images was performed using the easy Z-score imaging system (eZIS) [3] and voxel-based stereotactic extraction estimation (vbSEE) software programs [4]. Hypoperfused regions were mainly observed in the right hemisphere, such as the pre- and postcentral gyri, superior and inferior parietal lobules, supramarginal gyrus, angular gyrus, superior, middle, and inferior temporal gyri, and superior and middle frontal gyri (Fig. 1b).

The general neuropsychological assessments performed were the MMSE, the Kohs Block test, WAIS-III, Wechsler Memory Scale-Revised, Behavioral Assessment of the Dysexecutive Syndrome, the Trail Making test, Clinical Assessment for Attention, and the Behavioral Inattention test, the results of which were all within normal ranges.

- The tactile recognition in the upper extremities was assessed 40 days after the onset of cerebral infarction.

- The elementary sensations for pain, temperature, light touch, vibration, and position sensations were all preserved.

- The discriminative sensations for topographic sensation (tactile localization) in 19 body parts were correct for 19/19 points on the right side and 7/19 on the left side. The 2point discrimination using the Spearman esthesiometer was $3 \mathrm{~mm}$ at the tips of the fingers of both hands; no tactile distinction was observed. 
- Hylognosia (perception of weight, texture, and quality of materials) was assessed by requesting the blindfolded patient to feel different stimuli in each hand.

Weight discrimination was assessed using 3 boxes of equal size, weighing 50, 56, and $62 \mathrm{~g}$. The patient was asked to arrange the boxes by weight (lightest to heaviest). Three trials were performed for each hand, and the patient was able perform the task correctly with both hands. Texture discrimination was assessed by presenting the patient with 3 types of sandpaper of Japanese Industrial Standards (JIS) 600 (maximum particle size $<72 \mu \mathrm{m}$ ), 800 (maximum particle size $<67 \mu \mathrm{m}$ ), and 1,000 (maximum particle size $<63 \mu \mathrm{m}$ ). Three trials were performed for each hand, and the patient was able to perform the task correctly with both hands.

Discrimination of the quality of materials was assessed by presenting the patient with 5 kinds of material: metal, wood, plastic, sponge, and cloth. Ten trials were performed for each hand. The patient was able to discriminate the quality of materials with no errors with both hands.

Morphagnosia (perception of size and shape) was assessed by requesting the blindfolded patient to feel different stimuli in each hand. Size discrimination was assessed with a wooden bar of 4 lengths $(2,4,6$, and $8 \mathrm{~cm})$ with a diameter of $1 \mathrm{~cm}$. The patient was asked to align the bars by length (shortest to longest). Three trials were performed for each hand, and the patient was able to perform the task correctly with both hands. Discrimination of 2dimensional shapes was assessed using 6 palm-sized figures made of cardboard with a thickness of $0.2 \mathrm{~mm}$ (square, circle, triangle, star, pentagon, and hexagon). Ten trials were performed for each hand, and the number of correct answers was as follows: 10/10 in the right hand and 3/10 in the left hand. The response time was 2-3 s in the left hand and 10-15 $s$ in the right hand, and the patient correctly identified the circle and square. Discrimination of 3-dimensional shapes was assessed using 5 palm-sized figures made of wood (cube, globe, cuboid, column, and triangular pyramid). Ten trials were performed for each hand and the number of correct answers was as follows: 10/10 in the right hand and 2/10 in the left hand. The patient only correctly identified the globe in the left hand.

Recognition of real objects was assessed using 15 household utensils (toothbrush, pencil, eraser, candle, scissors, glasses, stapler, fork, button, wrist watch, comb, nail clippers, cup, iron nail, hammer, and ballpoint pen). The patient correctly recognized all of the real objects (15/15 in both the right and left hands). The recognition of objects by the right hand was complete and immediate (within 2-3 s), but the response for the left hand took 10-15 s, particularly for the pencil, ballpoint pen, and candle, which were similar in shape and size. Regarding the scissors, the patient commented that they felt cold and finally recognized that. Regarding the fork, she commented that it had a sharp end, and then recognized the fork; these statements suggest that the patient was using the information from hylognosia and size to identify the objects. After the recognition trials for the real objects with the left hand, the patient was asked to draw the tested objects to evaluate whether or not she could recognize the items; she was able to draw all test items (Fig. 1c).

\section{Discussion}

The present patient showed unilateral amorphagnosia limited to 2- and 3-dimensional figures that was not attributable to impaired sensation. Notably, her perception of the weight, texture, and quality of materials and the tactile recognition of real objects were not impaired. Saetti et al. [5] proposed a schematic model of tactile recognition (Fig. 2). There 
have been 5 reports (6 cases) on apperceptive agnosia and tactile agnosia [6-9]. Four cases reported by Bohlhalter et al. [7], Crutch et al. [8], and Estrafiol et al. [9] showed defects in both hylognosia and morphagnosia (Fig. 2; routes A, B, and C) which resulted in tactile agnosia. The patients reported by Reed et al. [6] showed amorphagnosia and mild ahylognosia, as well as tactile agnosia. The case of Saetti et al. [5] showed both tactile agnosia and amorphagnosia confined to the shape (Fig. 2; route C); the patient was unable to identify the shape of the stimulus which was associated with a striking impairment in detecting the orientation of a line or a rod in 2- and 3-dimensional space. This spatial deficit was thought to underlie amorphagnosia, since recognition of the tactile modality form is built upon the integration of the successive changes in orientation in space made by the hand as it explores the stimulus. The patient had a lesion located in the right hemisphere that encroached upon the posterior parietal cortex, which is the region presumed to specialize in shape recognition. Valenza et al. [10] reported a case of left unimanual impairment in tactile object recognition. The patient showed a dissociation between active and passive shape recognition, demonstrating a deficiency in active exploratory movement for shape recognition. The authors concluded that the patient had pure tactile apraxia. However, none of the previously reported patients, including our case, exhibited the dissociation between active and passive recognition as found in Valenza's patient.

The identification of objects by touch is a complex and poorly understood process. The mechanisms involved in tactile object recognition and the anatomical correlation with these mechanisms have not been precisely defined.

Bohlhalter et al. [7] and Burton et al. [11] proposed that the perception of weight, texture, and material quality (hylognosia) is mainly impaired due to lesions in the primary sensory cortex (SI), whereas the perception of size and shape (morphagnosia) is impaired due to lesions in the secondary somatosensory area (SII) and the posterior parietal cortex in a parallel processing fashion. However, Estrafiol et al. [9] reported a case of tactile agnosia with impaired hylognosia_and morphagnosia due to a lesion confined to the primary sensory cortex (S1) in the right parietal hemisphere, sparing other regions of the cerebral cortex which presumably participate in tactile object recognition.

Using functional MRI in healthy subjects, Stoeckel et al. [12] showed that the right superior parietal cortex is specifically activated by kinesthetic attention during tactile object discrimination. The lesions in our case were present in the right postcentral gyrus and subcortical area of the middle frontal gyrus according to MRI. The analysis of SPECT images using the eZIS and vbSEE software programs revealed that the greatest reductions were localized to the pre-and postcentral gyri, superior and inferior parietal lobules, supramarginal gyrus, and angular gyrus.

Concerning the side of the lesions, the cases reported by Reed et al. [6], Bohlhalter et al. [7], and Crutch et al. [8] were all localized to the left hemisphere. Accordingly, the lesions responsible for the impaired hylognosia and morphagnosia may not necessarily be confined to the right hemisphere.

The mechanisms involved in tactile object analysis and the anatomical correlates of those mechanisms have not been precisely defined. The tactile agnosia observed in the cases of Bohlhalter et al. [7], Crutch et al. [8], and Estrafiol et al. [9] may have resulted from the defects in both hylognosia and morphagnosia (apperceptive tactile agnosia).

Our case is unusual in that the patient showed unilateral amorphagnosia limited to the 2- and 3-dimensional figures in the left hand, but she had neither hylognosia nor tactile recognition of real objects. While the recognition of objects by the right hand was complete and immediate, the recognition by the left hand was slow. However, the slow response was 
not associated with agnosia of these real household objects. We considered the mechanism for the well-preserved recognition of real household objects as follows: the repeated exploratory action of the objects by the left hand makes it possible to obtain information regarding the objects using the intact hylognosia (route A) and size perception (route B, one of the routes of morphagnosia), despite the absence of any information from the morphagnosia for 2- and 3-dimensional shape perception (route $\mathrm{C}$ ), and this could thus result in the recognition of real objects.

The frequency of the correct recognition of real objects differs among the reported papers; for example, the correct recognition rate of Reed's case, who showed amorphagnosia and mild ahylognosia, was 13/28 objects (46.2\%), while Saetti's case, who showed amorphagnosia only, recognized $7 / 10$ objects $(70 \%)$.

These present and previous findings suggest that the mechanism for the well-preserved recognition of real objects may depend on the preserved function of the hylognosia. However, there have been no reports showing only ahylognosia without amorphagnosia. Further studies are necessary to clarify whether or not patients with preserved hylognosia or morphagnosia retain the ability to perceive real objects.

\section{Statement of Ethics}

Informed consent was obtained from the patient on November 19, 2012.

\section{Disclosure Statement}

The authors have no relevant financial or nonfinancial relationships to disclose.

\section{References}

1 Wernicke C: Zwei Faelle von Rindenlaesionen. Arb Psychiatr Klin Breslau 1895;2:33-55.

2 Delay J: Les Astereognosies. Pathologie du Toucher. Paris, Masson \& Cie, 1935.

-3 Kanetaka H, Matsuda H, Asada T, et al: Effect of partial volume correction on discrimination between very early Alzheimer's dementia and controls using brain perfusion SPECT. Eur J Nucl Med Mol Imaging 2004;31:975-980.

4 Mizumura S, Kumita S, Cho K, et al: Development of quantitative analysis method for stereotactic brain image; assessment of reduced accumulation in extent and severity using anatomical segmentation. Ann Nucl Med 2003;17:289-295.

-5 Saetti MC, Renzi ED, Comper M: Tactile morphagnosia secondary to spatial deficits. Neuropsychologia 1999;37:1087-1100.

-6 Reed CL, Caselli RJ, Farah MJ: Tactile agnosia underlying impairment and implications for normal tactile object recognition. Brain 1996;119:875-888.

7 Bohlhalter S, Fretz C, Weder B: Hierarchical versus parallel processing in tactile object recognition. Brain 2002;125:2537-2548.

8 Crutch S, Warren JD, Harding L, Warrington EK: Computation of tactile object properties requires the integrity of praxic skills. Neuropsychologia 2005;43:1792-1800.

-9 Estrafiol B, Baizabal-Carvallo JF, Senties-Madrid H: A case of tactile agnosia with a lesion restricted to the postcentral gyrus. Neurol India 2008;56:471-473.

10 Valenza N, Ptak R,Zimine I, Badan M, Lazeyras F, Schnider A: Dissociated active and passive tactile shape recognition: a case study of pure tactile apraxia. Brain 2001;124:2287-2298.

11 Burton H, Sinclair RJ: Attending to and remembering tactile stimuli: a review of brain imaging data and single-neuron responses. J Clin Neurophysiol 2000;17:575-591.

12 Stoeckel MC, Weder B, Binkofeki F, Choi HJ, Amunts K, Pieperhoff P, et al: Left and right superior parietal lobule in tactile object discrimination. Eur J Neurosci 2004;19:1067-1072. 


\section{Case Reports in Neurology}
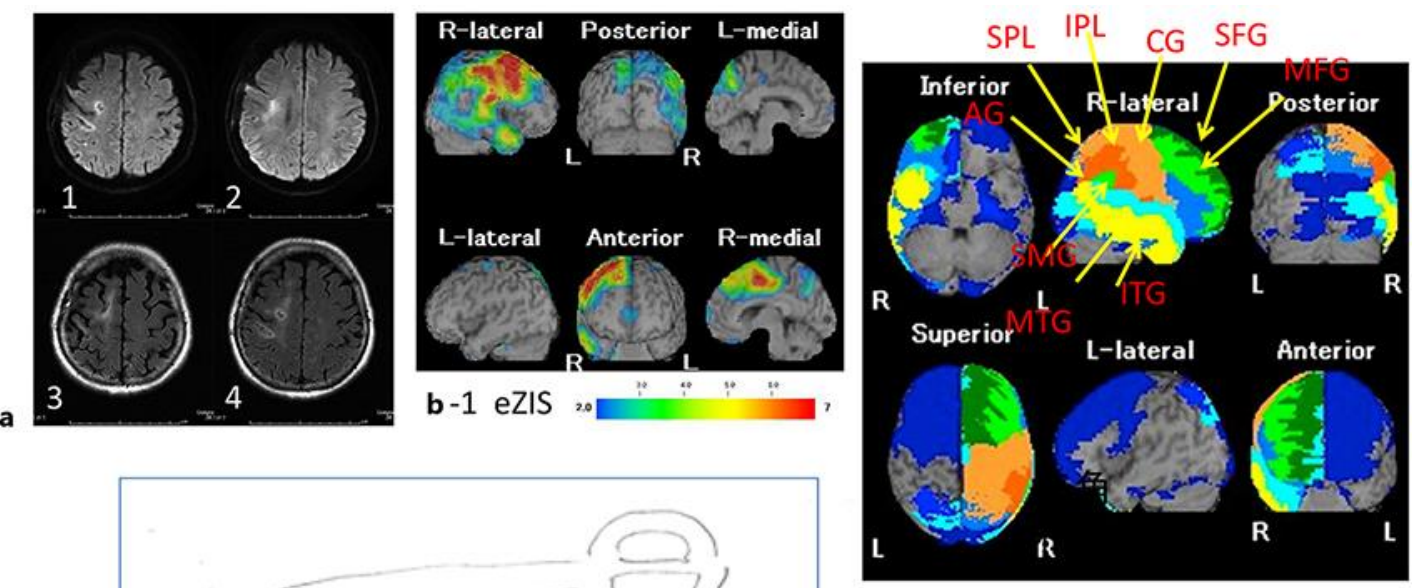

b-2 VbSEE

scissors
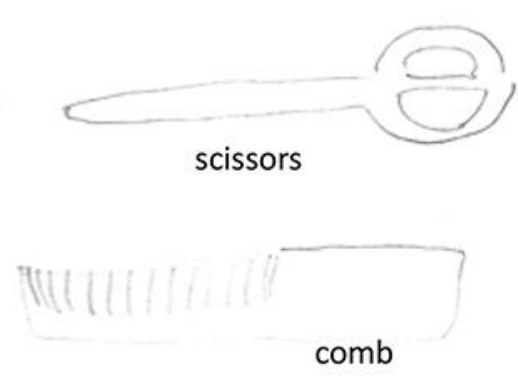

c

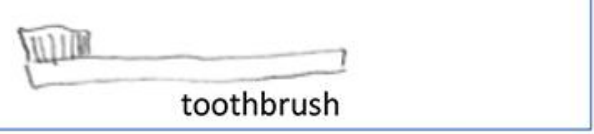

Fig. 1. a DWI (images 1 and 2) and FLAIR (images 3 and 4) axial images showing the hyperintense lesions in the right postcentral gyrus, precentral gyrus, and medial frontal cortex. b eZIS image (b-1) showing hypoperfusion in the right frontoparietal areas. vbSEE image (b-2) showing the hypoperfusion regions mainly in the right hemisphere such as the pre-and postcentral gyri (CG), superior and inferior parietal lobules (SPL, IPL), supramarginal gyrus (SMG), angular gyrus, middle and inferior temporal gyri (MTG, ITG), and superior and middle frontal gyri (SFG, MFG). c Figures showing samples of the real objects drawn by the patient after the trials of recognition of real household utensils. 


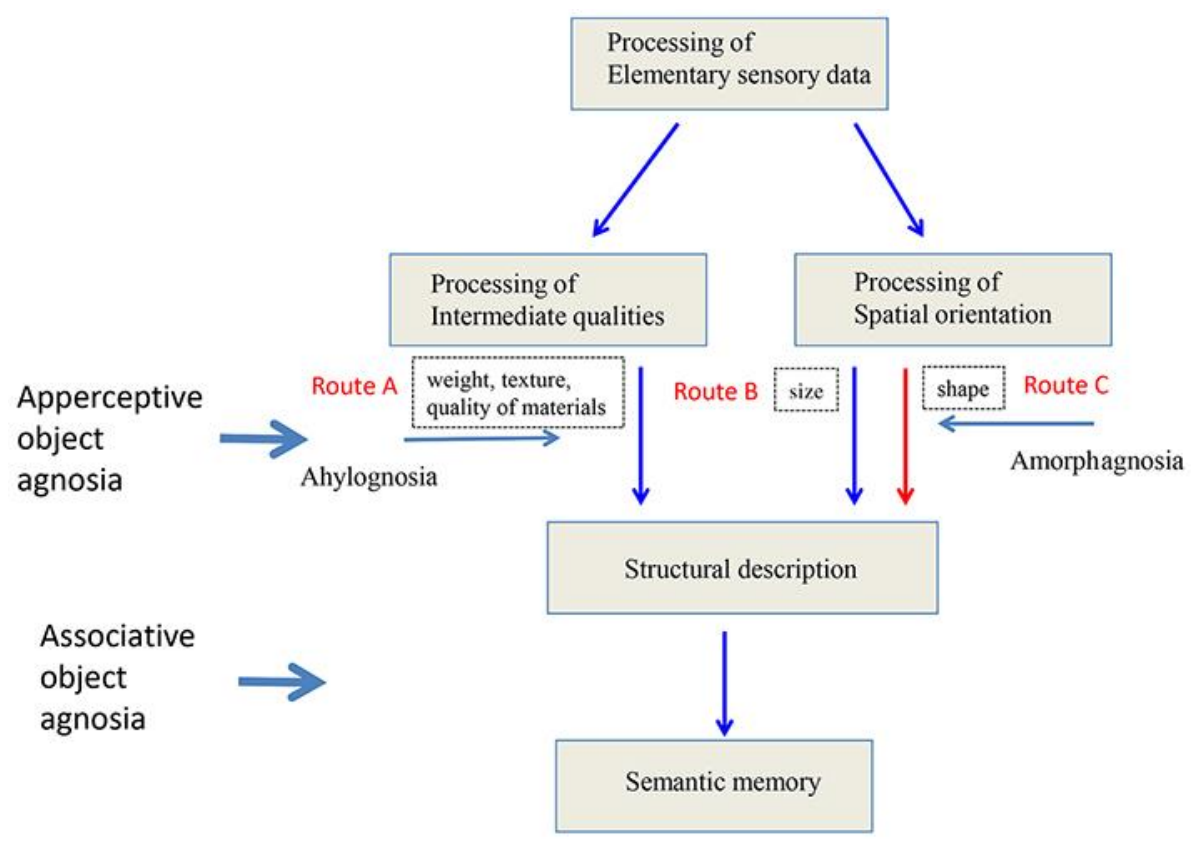

Fig. 2. A modified schematic model of tactile recognition by Saetti et al. [5]. 